\title{
ALTERATION OF ACTIVITY OF SINGLE NEURONS IN THE NUCLEUS CENTRUM MEDIANUM FOLLOWING STIMULATION OF THE PERIPHERAL NERVE AND APPLICATION OF NOXIOUS STIMULI
}

\author{
Miyoshi Urabe, Takashi TsuboKawa \\ AND Yoh WATANABE \\ Department of Surgery, School of Medicine, University of Kanazawa
}

It has been known that the nucleus centrum medianum $(\mathrm{CM})$ in the thalamus had functions as a termination of the afferent fibers through the anterolateral tract in the spinal $\operatorname{cord}^{2,3,5,6)}$ as well as a relay nucleus of the afferent fibers of the extrapyramidal system from the cerebellum ${ }^{4}$. It has been also clarified that stimulation of the $\mathrm{CM}$ caused a recruiting response on the electrocorticogram and induced EPSP as well as IPSP in the pyramidal cell ${ }^{9}$. ALBE-FESSARD \& KRUGER ${ }^{1)}$ demonstrated three kinds of action potential activated by stimulation of the peripheral nerve. URABE et al. ${ }^{10)}$ found that the evoked potentials were convergently elicited in the ventroposterior part of the $\mathrm{CM}$ by stimulation of the splanchnic or sciatic nerve. They observed also the spindle burst developed in the CM following the tetanic stimulation of the splanchnic nerve and noticed that the spindle burst was much influenced by the activities of the mesencephalic reticular formation (MRF) and the cortex ${ }^{11}$. And it was confirmed that these afferent transmissions belonged to the ascending system through the anterolateral tract. According to the investigation on the neuronal activity of the CM by URABE et al. $^{10,11)}$, the driven unitary discharge with an average latency of $18.0 \pm 1.5 \mathrm{msec}$ by sciatic stimulation was recorded from an identical neuron to the one where the driven unitary discharge with an average latency of $18.6 \pm 1.4 \mathrm{msec}$ by splanchnic stimulation was recorded. Interaction between the responses of the CM neuron by splanchnic and sciatic stimulations was recognized, as the testing response was inhibited, if conditioning and testing stimuli were applied at an interval of less than $60-120 \mathrm{msec}$. These phenomena indicate that a convergence of the splanchnic and sciatic transmissions exists in the CM-neurons. Furthermore, it was clarified that the CM-neuron was activated to volley the potential by noxious stimuli.

Received for publication December 26, 1965

卜部美代志, 坪川孝志, 渡辺洋宇 
In the present paper, the functional contribution of the CM-neuron to perception of the sensory information will be discussed by means of investigation of the CM-neuron which was endowed with such characteristics as convergence of the splanchnic and sciatic transmissions and response to noxious stimuli.

\section{METHODS}

The experiment was performed on 36 adult cats. Surgical procedures were done under ether anesthesia. All wound margins and pressure areas were infiltrated with $1 \%$ procaine. When the anesthetic effect of ether disappeared, subsequent observations were done after immobilization of the animal with 0.05 to $0.1 \mathrm{mg} / \mathrm{kg}$ of hexamethylens1.6-bis-carbaminoxylcholine bromide (Carbogen), respiration being maintained artificially through a tracheal cannula.

The splanchnic nerve was exposed by the extrapleural or intrathoracic approach. The sciatic nerve was exposed on the hindlimb. A bipolar silver wire was hooked to the central end of the severed peripheral nerve. Square pulse stimulation was applied with 10 volts for a duration of $1-5 \mathrm{msec}$. Tetanic stimulation of the splanchnic nerve was delivered at a frequency of $10 \mathrm{cps}$.

After fixation of the head of the animal on the stereotaxic apparatus, three burr holes of $3 \mathrm{~mm}$ diameter were drilled for the introduction of the tungsten microelectrodes to the $\mathrm{CM}$ and for insertion of the stimulating concentric bipolar electrodes into the MRF and contralateral CM. Electrical stimulation through these bipolar electrodes was done with a square pulse of 4 volts, $0.5 \mathrm{msec}$ duration and 10 or $100 \mathrm{cps}$ frequency. The unitary discharge of the $\mathrm{CM}$ was picked up with tungsten microelectrodes of $1-3 \mu$ diameter coated with Envy $\$ 1000$. Continuous recording was done with a cathode follower (12 AU 7) and by photographying the beam with a 2-channel oscilloscope ( $\mathrm{VC}-7$ ).

The recording microelectrodes for action potential were inserted stereotaxically according to JASPER \& AJMONE-MARSAN's atlas. The CM-neuron was identified in the following way. The first prerequisite for the CM-neuron was to establish whether or not the spontaneous unitary discharges were facilitated by tetanic electrical stimulation of both the splanchnic and sciatic nerves. The second prerequisite for the CMneuron was to establish whether or not the driven unitary discharges were convergently recorded from a single neuron by stimulation of the splanchnic as well as sciatic nerves and an interaction between the responses of the CM-neuron by splanchnic and sciatic stimulations. The third prerequisite for the CM-neuron was to establish whether or not the neuronal action potential was obtained responding to natural noxious stimuli applied to the skin on more than two limbs (FIG. 1).

Natural stimuli included a light scrub of the hair or skin with a hair brush, mechanical stimulation of the skin with a blunt object such as a nail or pencil eraser and presumably noxious stimuli produced by heating or pinching the tissue between the fingers or pricking to drop a needle with $10 \mathrm{gr}$ weight on the skin. The location of the cutaneous receptive field was determined by observing the potential response to noxious stimuli on the skin where the site was marked.

After recording the $\mathrm{CM}$ neuron's activity, a small electrolytic lesion was made at the bottom of each track to aid in the identification of the microelectrode position. At the conclusion of each experiment, the cat was killed by an overdose of Nembutal and perfused with saline then with a neutral $10 \%$ formalin-saline solution. The brain was taken out and fixed with a $10 \%$ formalin solution for 10 days, then, in serial sections 

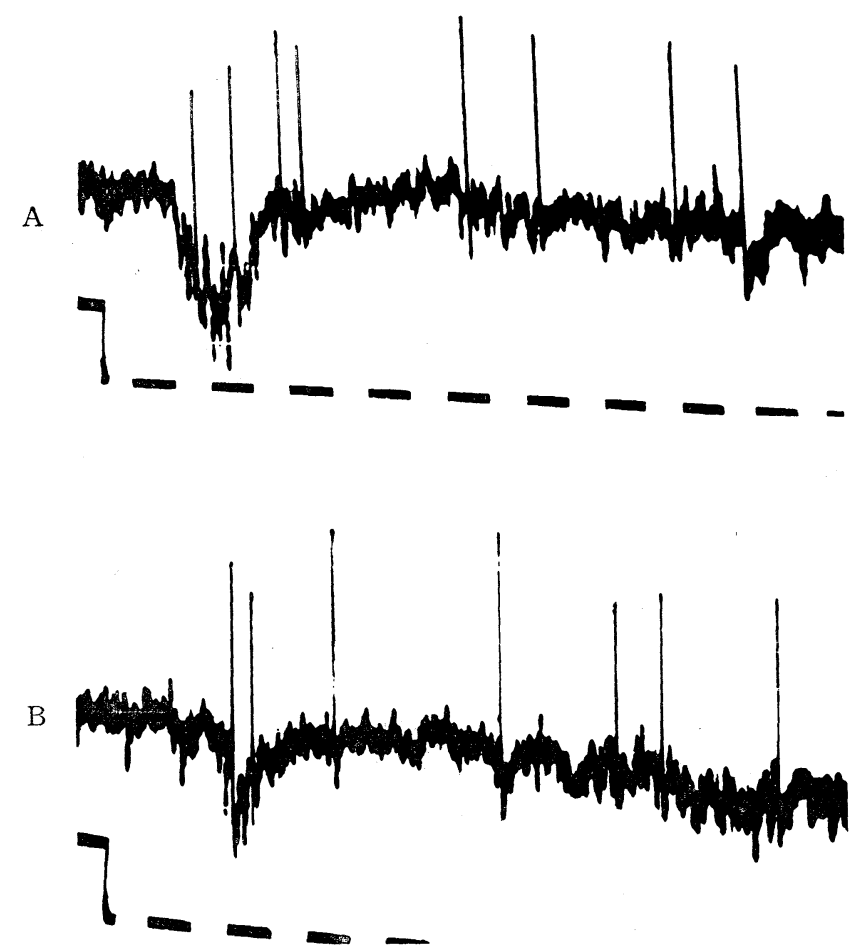

\section{$10 \mathrm{msec}$.}

FIG. 1. Identification of the CM-neuron. The prerequisite for the CM-neuron was to establish that driven unitary discharge were recorded from a neuron in response to stimulation of the sciatic nerve (A) as well the splanchnic nerve (B) and that the neuron responded to noxious stimuli having large receptive field which covered more than two limbs. Downward deflection: positive phase. Stimulation for $5 \mathrm{msec}$.

of $50 \mu$ on the stereotaxic plane and stained with a $0.1 \%$ thionine solution for verification of the electrode position.

\section{RESULTS}

1) Patterns of the spontaneous unitary discharge of the CM-neuron. The CMneurons volleyed the spontaneous unitary discharge with a frequency of about 5 to $27 / \mathrm{sec}$. The interval histogram produced from the 100 spontaneous firings is shown in Fig. 2. The average interval was $34.1 \mathrm{msec}$ with a standard error of $13.8 \mathrm{msec}$. A few large deviations were noticed. No burst-like volley was seen in the spontaneous discharge of all the CM-neurons. As related by URABE et al. ${ }^{11}$ in the other paper, the spontaneous unitary discharge of the CM-neuron was inhibited to decrease the number of discharges and came to 


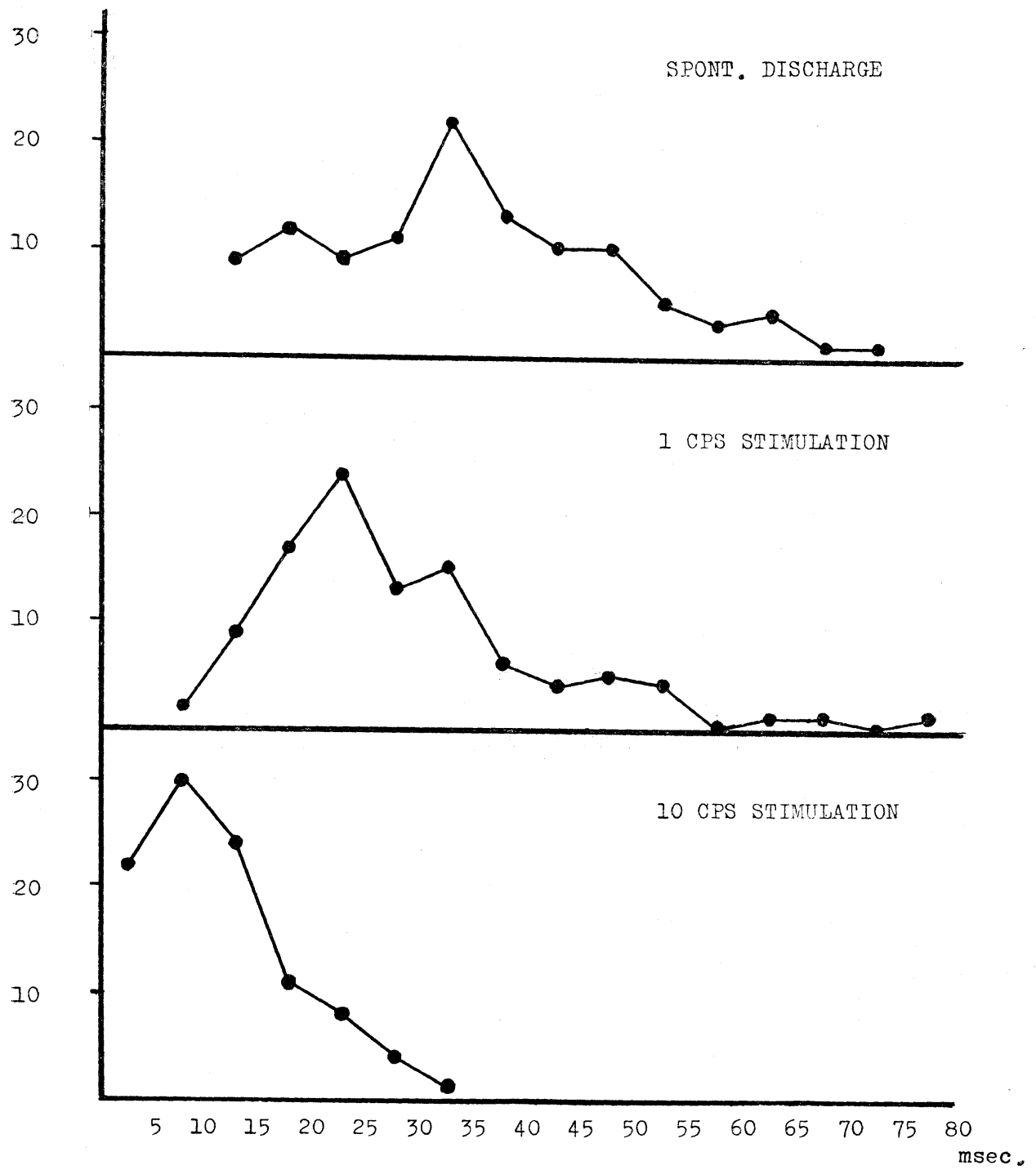

FIG. 2. Interval histogram of the spontaneous firing of the CM-neurons in response to stimulation of the splanchnic nerve. From top to bottom. Spont. Discharge: Spontaneous unitary discharges before stimulation of the peripheral nerve. Interval histogram produced with 100 firings. $1 \mathrm{cps}$ Stimulation: Spontaneous unitary discharges during stimulation of $1 \mathrm{cps}$. $10 \mathrm{cps}$ Stimulation: Spontaneous unitary discharges during stimulation of $10 \mathrm{cps}$. Vertical axis: number of unit. Horizontal axis: firing interval.

show a grouped pattern under Nembutal anesthesia. And the spontaneous unitary discharge was facilitated by brief train stimulation of the MRF and contralateral $\mathrm{CM}$ with a frequency of $10 \mathrm{cps}$ or $100 \mathrm{cps}$, also, it was facilitated 
following the cortical strychnization ${ }^{11}$. In the present investigation, facilitation of the spontaneous unitary discharge in the CM following tetanic stimulation of the splanchnic nerve was seen in the same way as in the case of the cortical strychnization, though the driven unitary potential responding to the splanchnic nerve stimulus failed to discharge. The interval histogram produced with the 100 spontaneous firings showed that after $1 \mathrm{cps}$ stimulation of the splanchnic nerve the interval between spikes shortened, being $28.2 \mathrm{msec}$ on the average and the number of firings increased with two peaks at the interval of $22.5 \mathrm{msec}$ and $32.5 \mathrm{msec}$. It was also exhibited that after $10 \mathrm{cps}$ stimulation of the splanchnic nerve, the interval shortened much more markedly, being $11.1 \mathrm{msec}$ on the average and the number of firings increased with a peak at the interval of $7.5 \mathrm{msec}$ showing a regularly changed pattern in firing.

When stimulation of $1 \mathrm{cps}$ was repeatedly applied to the sciatic nerve with 30 trials, the driven unitary potential and succeedingly developed spontaneous firings in the CM-neuron or in the neuron of the nucleus ventralis posterolateralis (VPL) were observed in every $50 \mathrm{msec}$ for $1 \mathrm{sec}$. In the CM-neuron, the firings for the first $50 \mathrm{msec}$ contained all of the driven unitary potential and some of the spontaneous discharge and they increased to 3 times that of the ordinary firing rate. From the next $100 \mathrm{msec}$, the firings which contained only the spontaneous potential continued to increase until $550 \mathrm{msec}$. In the VPL-neuron, the firings for the $50 \mathrm{msec}$ contained all of the driven unitary discharge and increased as well. But from the next $100 \mathrm{msec}$ to $250 \mathrm{msec}$ the firings containing the spontaneous discharge were inhibited to decrease the frequency (FIG. 3). Thus, the driven unitary discharge activated by $1 \mathrm{cps}$ stimulation of the sciatic nerve, induced facilitation of the spontaneous firing in the CM-neuron for $550 \mathrm{msec}$ while it induced inhibition of the spontaneous firing in the VPL-neuron for $250 \mathrm{msec}$.

As related above, the driven unitary discharge in the $\mathrm{CM}$ failed to respond to an individual stimulus when brief train stimulation was applied to the splanchnic or sciatic nerve, while the spontaneous unitary discharge was facilitated following stimulation. And URABE et al. ${ }^{10)}$, had found in the observation of the slow potential that the evoked potential in the $\mathrm{CM}$ did not follow an individual stimulus and the spindle burst appeared in the CM when brief train stimulation was applied to the splanchnic nerve. In relation to the above, the facilitation of the activity of the CM-neuron in 63 units following $10 \mathrm{cps}$ stimulation of the splanchnic nerve was investigated. Neurons in $16 \%$ of 63 units showed a marked increase in spontaneous unitary discharge during tetanic stimulation of $10 \mathrm{cps}$. After tetanization, the spontaneous unitary discharge continued to increase in firing for 5 to 15 seconds while being synchronized to the appearance of the spindle burst of the slow potential (FIG. 4-A). Neurons in $43 \%$ showed a continuous marked facilitation in spontaneous 


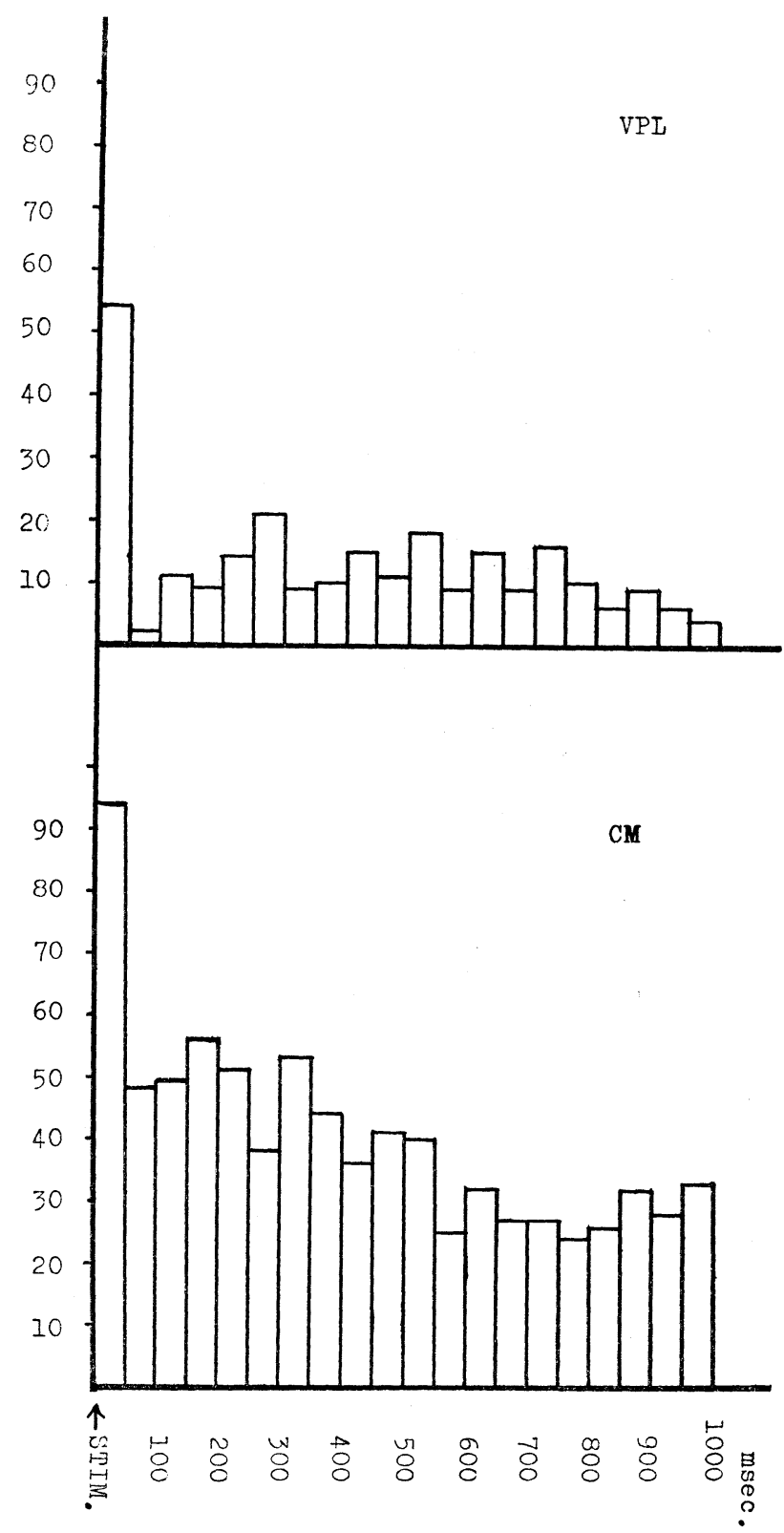

FIG. 3. The firing number in every $50 \mathrm{msec}$ for $1 \mathrm{sec}$ when $1 \mathrm{cps}$ stimulation was applied to the sciatic nerve with 30 trials. VPL: The firing pattern in the VPL-neuron. The firings from the second $50 \mathrm{msec}$ contained the spontaneous discharge and they were inhibited for $250 \mathrm{msec}$. CM: The firing pattern in the CMneuron. The firings from the second $50 \mathrm{msec}$ contained the spontaneous firing and they continued to increase for $550 \mathrm{msec}$. 


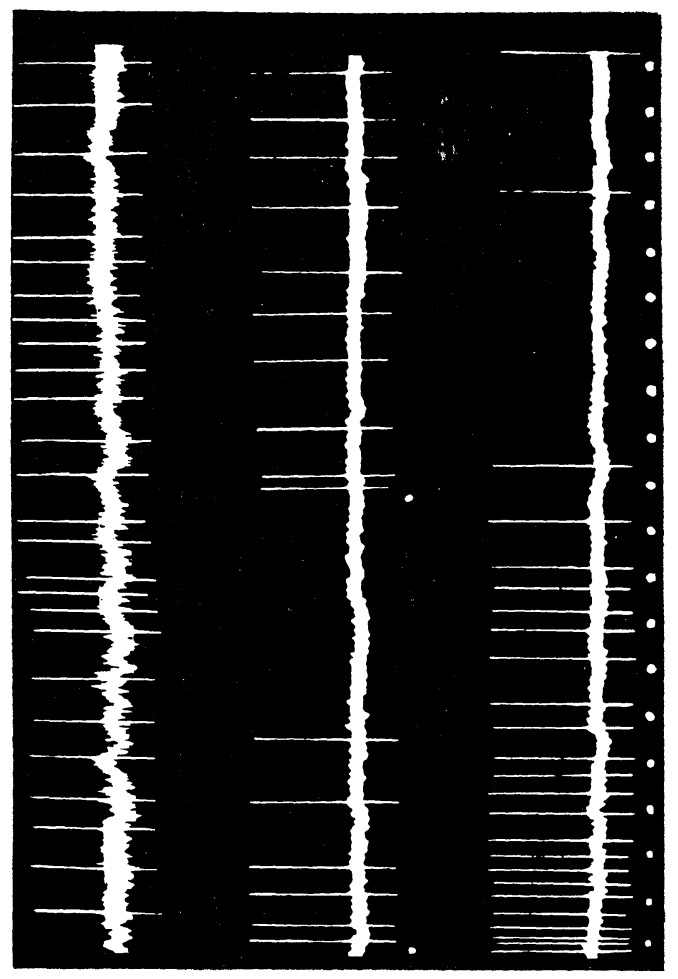

p

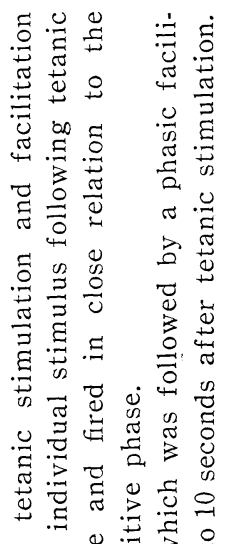

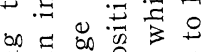

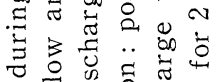

○

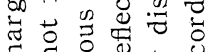

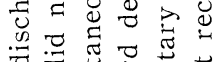

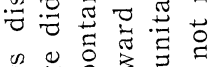

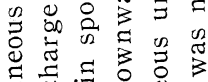

芯总.

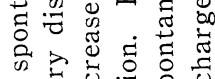

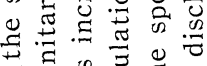

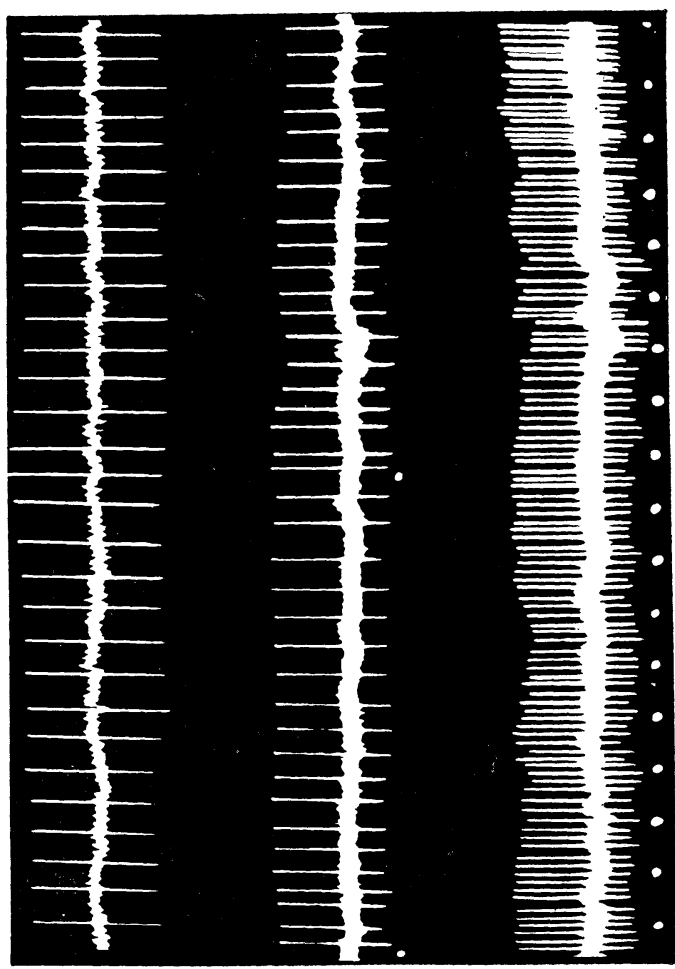

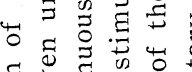

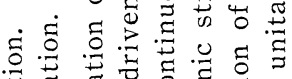

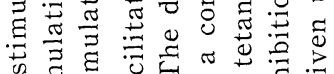

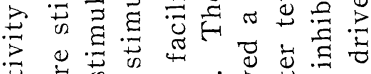

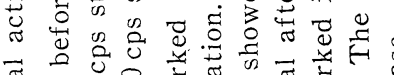
สี

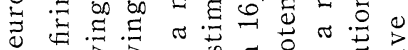

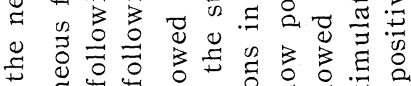

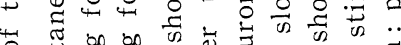

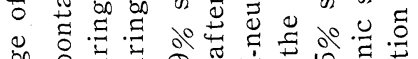

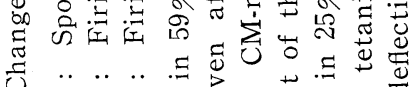

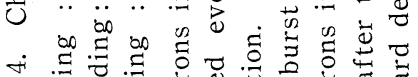
ن

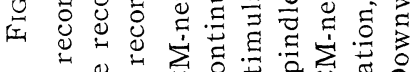
- 仓 
unitary discharge lasting even after tetanization, but without appearance of the spindle burst. In these cases, however, it was because of electrode resistance that the slow potential was not recorded simultaneously with the unitary potential. Facilitation of these neurons might occur in the same modus as in the first group. Neurons in $25 \%$ showed a marked inhibition of the spontaneous unitary discharge during and shortly after tetanic stimulation. Inhibition was followed by a phasic facilitation for 3-5 seconds, then, inhibition continued. The driven unitary discharge was not recorded for 2 to 10 seconds after tetanic stimulation (FIG. 4-B). Neurons in $16 \%$ showed a variable facilitation in spontaneous unitary discharge after tetanization.

These phenomenon in the CM-neuron following tetanic stimulation of the splanchnic nerve were different from those in the VPL-neuron following the same conditioning. These phenomena were rather similar to those in the MRF-neuron. However, there was no neuron in the MRF which showed characteristics as in the first and third groups.

2) Action potential of the CM-neuron in response to electrical stimulation of the splanchnic or sciatic nerve. The driven unitary discharge by stimulation of the unilateral peripheral nerve was picked up from the CM-neuron on the bilateral sides. The discharge was not obtained after transection of the anterolateral tract, though it remained without change after transection of the dorsal funiculus.

The latency of the driven unitary discharge following stimulation of the splanchnic nerve was observed in 29 units of the CM. It ranged from 16.7 to $21.9 \mathrm{msec}$. The average latency was $18.7 \mathrm{msec}$ with a standard error of 1.4 msec. The driven unitary discharge with a long latency as ALBE-FESSARD \& KRUGER $^{1)}$ observed, was not recognized in the present investigation. Observing the number of discharge per stimulus, one firing was seen in $24.4 \%$ of all the units, two firings in $40.4 \%$, three firings in $32.9 \%$ and four firings in $2.4 \%$. The driven unitary discharge following stimulation of the sciatic nerve was also observed in the same 29 units of the CM as those which were the object of study by splanchnic stimulation. Its latency ranged from 15.0 to $20.0 \mathrm{msec}$ and the average latency was $18.0 \mathrm{msec}$ with a standard deviation of $1.5 \mathrm{msec}$. This latency was slightly shorter than in the case of stimulation of the splanchnic nerve. The units with two or three firings per stimulus amounted to $75.6 \%$ of all the units encountered.

An interaction between responses to splanchnic and sciatic stimulations was definitely recognized if the conditioning and testing stimuli were applied at an interval of less than $120 \mathrm{msec}$ (FIG. 5).

When tetanic stimulation with a frequency of more than $10 \mathrm{cps}$ was applied to the peripheral nerve, the driven unitary discharge in the $\mathrm{CM}$ did not follow an individual stimulus and it disappeared for 2-15 sec after tetani- 
A
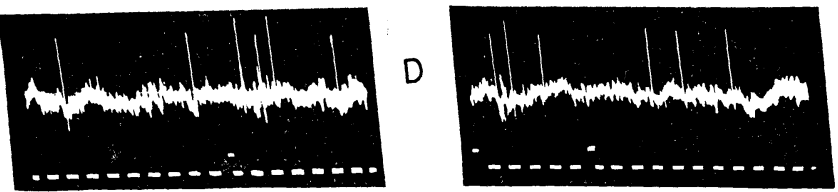

B
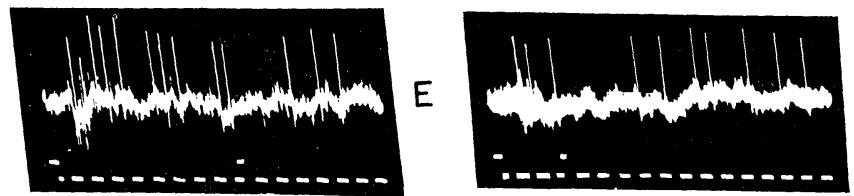

C

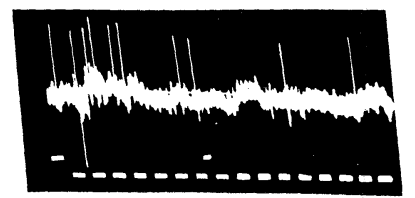

$\overline{50 \text { MSEC }}$

FIG. 5. Interaction between the impulses from the splanchnic nerve and the sciatic nerve. A: Unitary discharge of the CM-neuron to splanchnic stimulation alone. B: Testing stimulus to the splanchnic nerve given at an interval of 100 msec after a conditioning stimulus to the sciatic nerve. $\mathrm{C}$ : Interval from conditioning to testing stimulus: $80 \mathrm{msec}$. D : Interval from conditioning to testing stimulus: $60 \mathrm{msec}$. E: Interval from conditioning to testing stimulus: $40 \mathrm{msec}$. Note occlusion of the test response in the case of intervals less than $100 \mathrm{msec}$ between two stimuli. (cat, No. 55)

zation. On this occasion, the spontaneous unitary discharge in the CMneuron showed a great increment in frequency. It is characteristic that the driven unitary discharge in the $\mathrm{CM}$ was suppressed while the spontaneous firing was facilitated.

URABE et al. ${ }^{10,11)}$ had already reported that in the VPL-neuron following tetanic stimulation of the peripheral nerve, the driven unitary discharge completely followed an individual stimulus so far as stimulation was applied with a frequency of less than $40 \mathrm{cps}$, while the spontaneous unitary discharge was extremely inhibited.

3) The modality properties of the CM-neuron responding to noxious stimuli. It was noted that responding to noxious stimuli was one of the properties of the $\mathrm{CM}$-neuron. In the present investigation, the unitary potential was observed in all 82 units of the CM-neuron responding to noxious stimuli such as pinprick on the limb skin or pinching the tissue between the fingers. There was no CM-neuron which responded to gentle joint movement, touch, scrub of the hair or pressure on the skin. However, response to tactile stimulation came to be observed in $9 \%$ of the CM-neurons when the MRF was stimulated with a high frequency. 


$$
\text { 剖 }
$$


In 48 of the $82 \mathrm{CM}$-neurons, the peripheral receptive fields were determined by application of localized noxious stimuli of pinprick or pinch. The receptive field of the CM-neuron was very large and covered the part on both sides of the body extending to more than two limbs. Though the CM-neuron responded to noxious stimuli on the bilateral sides, the response was much more intensively seen from the stimuli on the contralateral side than the ones on the ipsilateral side and an increase in firing rate of action potential and a prolongation in duration of after-discharge were noted. The firing rate of action potential and the duration of after-discharge were different according to the site of the receptive field on one side (FIG. 6).

Observation of the CM-neuron led us to the concept that the CM-neuron. played an essential role in the neural mechanism of preception of noxious stimuli but has no function to discriminate distinctly the site of stimuli. Not

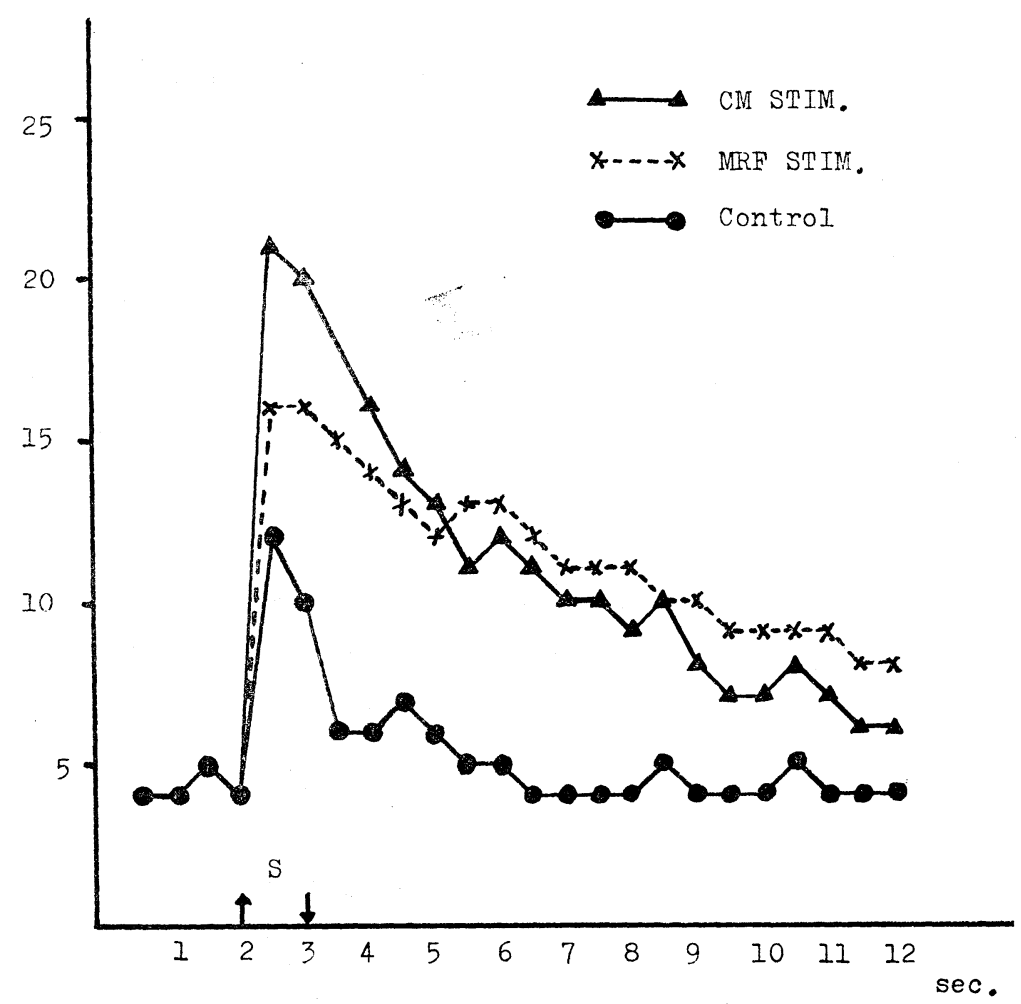

FIG. 7. Firing rate of the CM-neuron responding to pinprick stimulation on the contralateral fore $\operatorname{limb}(\mathrm{S})$. CM stim.: Response in the case of conditioning by $100 \mathrm{cps}$ stimulation of the contralateral CM. MRF stim.: Response in the case of conditioning by $100 \mathrm{cps}$ stimulation of the MRF. Control: Response before conditioning. Horizontal axis: time (sec). Vertical axis: discharge number per $0.5 \mathrm{sec}$. 
only the number of firings but also the duration of firings represented the intensity of stimuli applied.

URABE et al. ${ }^{11}$ had reported that the spontaneous unitary discharge increased in all units of the $\mathrm{CM}$ neuron after tetanic stimulation of the MRF or contralateral $\mathrm{CM}$ with either $10 \mathrm{cps}$ or $100 \mathrm{cps}$. In the present investigation, the response of the CM-neuron to pinprick on the limb skin was facilitated when the conditioning stimuli with a high frequency were applied to the MRF or contralateral $\mathrm{CM}$. The firing rate of the unitary potential in response to noxious stimuli more markedly increased and the duration of after-discharge became longer, than before conditioning stimulation (FIG. 7).

\section{DISCUSSION}

The function of the nucleus centrum medianum $(\mathrm{CM})$ in perception of noxious stimuli has not completely been understood, though some reports by Albe-Fessard \& KRUGeR ${ }^{1)}$, Whitlock \& PERL ${ }^{7,13)}$ and URABE et al. ${ }^{10,11)}$ have described this subject. AlBE-FEsSARD et al. ${ }^{1}$ found the CM-neuron which responded to pain sensation on the skin and differentiated two kinds of neurons according to the nature of the driven unitary discharge. But, URABE et al. ${ }^{10)}$ could not record the driven unitary discharge with a long latency in the CM as ALBE-FESSARD did. WhitLock \& PERL ${ }^{13)}$ found the neurons responding to noxious stimuli in the magnocellular part of the mediale geniculate body (MGmc) which corresponded to the neurons of the posterior nuclear group of the thalamus (PO) by PoGgio \& Mountcastle ${ }^{8}$. Disagreement among the views of many authors was caused by the variance of identification of the CM-neuron, difference of anesthesia and uncertainty in quantitative representation of noxious stimuli. In the present investigation, the prerequisite for the CM-neuron was to establish that the driven unitary potentials were recorded from a neuron by splanchnic as well as sciatic stimulation under unanesthetic condition and that the neurons responded to noxious stimuli with large receptive fields. The neurons which fulfilled this prerequisite were localized in the ventroposterior part of the CM where the present authors had recognized the spindle burst on the slow potential following tetanic stimulation of the splanchnic nerve.

It had been already reported by URABE et al. ${ }^{10)}$ that the spontaneous firing of the CM-neuron was facilitated, contrary to that of VPL-neuron, by tetanic stimulation of the MRF or contralateral CM. In the present investigation, the driven unitary discharge in the $\mathrm{CM}$ did not follow an individual stimulus but failed to volley and the spontaneous unitary discharge in the CM was facilitated, if the peripheral nerve was stimulated with an increasing frequency. This phenomenon in the CM-neuron was different from that in the VPL-neuron where the driven unitary discharge completely followed a stimulus 
and the spontaneous firing was inhibited after tetanic stimulation of the peripheral nerve. When the peripheral nerve was stimulated with a frequency of $1 \mathrm{cps}$, the spontaneous unitary discharge in the CM was facilitated for 550 msec, but the spontaneous unitary discharge which succeeded to the driven unitary discharge in the VPL was inhibited for $250 \mathrm{msec}$.

On this occasion, occurrence of depolarization in the CM-neuron for 550 msec might be explained if it was assumed that EPSP in the CM-neuron developed one after another for $550 \mathrm{msec}$ with a mediation of the impulse of a long latency which ascended the collaterals from the spinothalamic tract through the polysynaptic relays in reticular formations of the medulla oblongata, pons and mesencephalon. However, depolarization in the CM-neuron being continued, the driven unitary discharge should facilitate rather than fail to volley within $550 \mathrm{msec}$ after tetanic stimulation of the peripheral nerve. Therefore, the perception of noxious stimuli in the CM-neuron would not be completely explained by the mechanism of the driven unitary discharge through the spinothalamic tract or by the depolarization mechanism in the CMneuron induced by the impulse which was transmitted through the polysynaptic route of the collaterals from the spinothalamic tract in the reticular formations of the medulla oblongata, pons and midbrain.

The perception of noxious stimuli should be conducted in the following way being based on the present author's data that the spontaneous firing of the CM-neuron was facilitated by the cortical strychnization and stimulation of the MRF or contralateral CM, as reported before. The spontaneous firing of the CM-neuron was influenced by intrinsic activities in various cerebral regions. Peripheral noxious stimuli made the ascending impulse through the anterolateral tract in the spinal cord and caused the driven unitary discharge in the $\mathrm{CM}$ which induced the intensive spontaneous unitary firing of the neuron, being influenced by the polysynaptic central core conduction of activities in various cerebral regions, thus, noxious stimuli were perceived in the CM-neuron. This perception mechanism was indirectly supported by the fact that the action potential of the CM-neuron in response to noxious stimuli increased in firing rate and the after-discharge prolonged in duration if the conditioning of brief train stimulation was applied to the MRF or contralateral CM. It could be directly verified by the intracellular recording of the membrane potential of the CM-neuron.

\section{SUMMARY}

Using unanesthetized cats, investigation of the perception of the sensory information in the CM-neuron was conducted by microelectrodical recording of the spontaneous discharge of the neuron, the driven unitary discharge following stimulation of the peripheral nerve and the action potential of the neuron 
in response to noxious stimuli. The prerequisite for the CM-neuron was to establish that convergence of the splanchnic and sciatic transmissions existed in the neuron and that the neuron responded to noxious stimuli. The present authors have analysed 82 units of the CM-neuron which fulfilled the prerequisites. The results were summarized in the following way.

The spontaneous discharge of the CM-neuron had a frequency of 5 to 25 per second. The average interval showed $34.1 \mathrm{msec}$ with a standard deviation of $13.8 \mathrm{msec}$. The deviation was relatively large. The spontaneous discharge did not show a burst-like volley as far as the animal was in an unanesthetic condition. The interval of the spontaneous firing became shortened as the frequency increased in stimulation of the peripheral nerve. When stimulation of $1 \mathrm{cps}$ was applied to the peripheral nerve, the spontaneous discharge of the $\mathrm{CM}$ neuron succeeding from the driven unitary discharge increased in firing rate and was facilitated for $550 \mathrm{msec}$. When stimulation of $10 \mathrm{cps}$ was applied to the peripheral nerve, the driven unitary discharge of the CMneuron did not follow an individual stimulus but the spontaneous unitary discharge was extremely facilitated. The spontaneous firing of the CM-neuron was facilitated in various modes following tetanic stimulation of the splanchnic nerve. The neurons in 59\% showed a marked continuous facilitation of the spontaneous firing and some of these neurons showed a facilitation of the spontaneous firing in close relation to the spindle burst of the slow potential. And the neurons in 25\% showed a marked inhibition of the spontaneous unitary discharge followed by a phasic facilitation.

The receptive field of the CM-neuron responding to noxious stimuli was very large and covered the part on both sides of the body, extending to more than two limbs. The firing rate of action potential and the duration of after-discharge were different according to the site of the receptive field. The reactability of the $\mathrm{CM}$-neuron to noxious stimuli was augmented to increase the frequency of action potential and to prolong the duration of afterdischarge, following brief train stimulation of the MRF or contralateral CM by $10 \mathrm{cps}$ or $100 \mathrm{cps}$.

The perception of noxious stimuli was mostly related to the spontaneous discharge of the CM-neuron and supported by excitation in the polysynaptic central core conduction.

\section{REFERENCES}

1) Albe-Fessard, D. And Kruger, L.: Duality of unit discharge from cat centrum medianum in response to natural and electrical stimulation. J. Neurophysiol., 25 : 3-20, 1962.

2) Anderson, F. D. And Berry, C. M.: Degeneration studies of long ascending fiber system in the cat brain stem. J. Comp. Neurol., 111: 195-230, 1959.

3) Bowsher, D.: Termination of the central pain path in man; The conscious ap- 
plication of pain. Brain, $80: 606-622,1957$.

4) Hassler, R. ANd Jung, R.: The extrapyramidal motor system, Handbook of physiology, Section I, vol. II, Ed. J. FIELD, Washington, 1960.

5) Mehler, W. R.: The mammalian "Pain Tract" in phylogeny. Ann. Rec., 127? 332, 1957.

6) Mehler, W. R., Feferman, M. E. and Nauta, W. J. H.: Ascending axon degeneration following anterolateral chordotomy. Brain, 83: 718-750, 1960.

7) Perl, E.R. And Whitlock, D.G.: Somatic stimuli exciting spinothalamic projections to thalamic neurons in cat and monkey. Exp. Neurol., 3 : 256-296, 1961.

8) Poggio, G. F. And Mountcastle, V. B.: A study of the functional centribution of lemnical and spinothalamic system to somatic sensibility. Bull. Johns Hopkins Hosp., $106: 266-316,1959$.

9) Purpura, D. P. And Shofer, R. J.: Cortical intracellular potentials during augmenting and recruiting responses. J. Neurophysiol., 27 : 117-132, 1964.

10) Urabe, M., Tsubokawa, T., Watanabe, Y. and Kadoya, S.: Alteration of activity of the nucleus centrum medianum in the thalamus following the sensory stimulation. Jap. J. Physiol., 15: 28-41, 1965.

11) Urabe, M., Tsubokawa, T., Watanabe, Y. and Kadoya, S.: Driven and spontaneous activities of single neuron in the nucleus centrum medianum following stimulation of the peripheral nerve. Brain \& Nerve, 18:25-37, 1965 (Written in Japanese)

12) Urabe, M. And Tsubokawa, T.: Stereotaxic thalamotomy for the relief of intractable pain-CEM Thalamotomy-Tohoku J. Exp. Med., 85: 286-300, 1965.

13) Whitlock, D. C. And Perl, E. R.: Afferent projections of ventral funiculi to thalamus of cat. J. Neurophysiol., 22 : 133-148, 1959. 